\title{
GAYS Y LESBIANAS EN EL DEPORTE: DISCURSO DE JÓVENES UNIVERSITARIOS ESPAÑOLES EN TORNO A SU ACEPTACIÓN
}

\author{
GAYS AND LESBIANS IN SPORT. UNIVERSITY STUDENTS' \\ DISCOURSE ON THEIR ACCEPTANCE
}

\author{
GAYS E LÉSBICAS NO ESPORTE: O DISCURSO DE JOVENS \\ UNIVERSITÁRIOS ESPANHÓIS ACERCA DA SUA ACEITAÇÃO
}

Joaquín Piedra*

\begin{abstract}
Palabras clave:
Homofobia.

Sexualidad.

Deportes.

Adulto joven.

Deseabilidad social.

Resumen: La presencia de gays y lesbianas en el deporte es cada vez más notable. Los estudios realizados hasta el momento en países occidentales muestran un panorama diverso, desde una cierta normalización en determinadas sociedades hasta la persistencia de un clima hostil a la diversidad sexual. Ante la falta de estos estudios en España, el objetivo de este trabajo es describir el discurso usado por jóvenes universitarios españoles a la hora de explicar la presencia de gays y lesbianas en el deporte. Se emplea una metodología cualitativa mediante análisis de contenido a dos grupos de discusión en que participan nueve estudiantes universitarios. Los resultados muestran una cierta normalización inicial de gays y lesbianas en el deporte, más clara en el caso de las mujeres, pero apuntan a un rechazo latente y sutil que se manifiesta en cada una de las tres formas de homofobia (afectiva, cognitiva y conductual). En el deporte, los estereotipos sobre la homosexualidad generan rechazos en parte de la sociedad, y ésta ataca principalmente mediante la agresión verbal.
\end{abstract}

Abstract: The presence of gays and lesbians in sport is increasingly clear. Studies in Western countries show different scenarios, from certain normalization in some societies to a persistent hostile climate for sexual diversity. Given the absence of such studies in Spain, the aim of this paper is to describe the discourse used by Spanish university students to explain the presence of gays and lesbians in sport. It employs a qualitative methodology through content analysis of two focus groups with nine university students. The results show initial normalization of gays and lesbians in sport - clearer in the case of women - but point to a latent and subtle rejection that is apparent in each of the three forms of homophobia - affective, cognitive and behavioral. In sport, stereotypes about homosexuality create rejection by society, which attacks mainly through verbal aggression.

Resumo: A presença de gays e lésbicas no esporte tem sido cada vez mais notória. Estudos realizados em países ocidentais mostram panoramas diversos, desde uma certa normalização em determinadas sociedades até a persistência de um clima hostil à diversidade sexual. Considerando a ausência de estudos sobre essa temática na Espanha, o objetivo do presente artigo é descrever o discurso de estudantes universitários espanhóis sobre a explicação da presença de gays e lésbicas no esporte. No âmbito da metodologia qualitativa foram realizados dois grupos focais de nove estudantes cada e aplicada a análise de conteúdo aos dados coletados. Os resultados sugerem uma certa normalização inicial de gays e lésbicas no esporte, mais clara no caso das mulheres, mas apontam para uma rejeição latente e sutil expressa em cada uma das três formas de homofobia (afetiva, cognitiva e comportamental). No esporte os estereótipos sobre a homossexualidade geram rejeição por parte da sociedade, principalmente demonstrada por meio de agressões verbais.
*Universidad de Sevilla, Sevilla, España.

E-mail: jpiedra@us.es

Recebido em: 10-03-2015

Aprovado em: 23-07-2015

(c) (1) (8) Licence 


\section{INTRODUCCIÓN}

Hace ya años que toda la comunidad internacional reconoce y acepta los beneficios que reporta el deporte entre sus practicantes tanto a nivel fisiológico como psicológico, sociológico 0 educativo. Sin embargo, en determinados contextos y situaciones, el deporte puede ser fuente de discriminaciones, acoso y agresiones para determinadas personas (CLELAND, 2014). En este sentido, el deporte ha integrado tradicionalmente entre sus características no sólo las visiones estereotipadas de las masculinidades y las feminidades, sino que también los estereotipos ligados a la sexualidad (AYVAZO; SUTHERLAND, 2009, DOWLING, 2013). Estas relaciones de poder por el cual la sexualidad se reglamenta, y la heterosexualidad se institucionaliza, se denominan heteronormatividad, y convierten en "anti-natural" o tabú la homosexualidad y demás formas de sexualidad. Fruto de ello es que a pesar de que el colectivo homosexual lleva años luchando por eliminar las discriminaciones en la sociedad, sigue siendo un grupo claramente señalado y repudiado en algunos sectores o contextos de nuestra sociedad, tales como es el deportivo.

Según Anderson (2014), la trayectoria histórica del deporte en Occidente desde la Revolución Industrial ha ido identificándolo como un lugar de re-masculinización del hombre: el deporte se fue configurando como un terreno para eludir la feminización del hombre. Este proceso de configuración de la masculinidad en el deporte pasaba por rechazar y repudiar a aquellos hombres ( $\mathrm{y}$, en menor medida, mujeres) que no cumplían los requisitos: inclinación al uso de la violencia; homofóbico; estoicismo emocional; dispuesto a la lucha; separado de determinados deportes y actividades; y con contactos limitados entre hombres. La evolución de la sociedad occidental ha conferido un carácter más aperturista y respetuoso con la diversidad a algunas culturas.

Concretamente en el deporte, estos cambios sociales y políticos han permitido, como menciona Griffin (2012), que cada vez haya más deportistas abiertamente gays; que cada vez sean más los deportistas heterosexuales que defienden los derechos de gays y lesbianas; que exista una mayor concienciación de los técnicos. Algunas organizaciones comienzan a afrontar esta problemática en el deporte: existe una mayor información accesible para deportistas; incluso se comienzan a elaborar programas y guías para trabajar con este colectivo minoritario en el deporte. Sin embargo, la situación española, y la de otros muchos países, no es la misma. En nuestra sociedad prácticamente no existen figuras del deporte que públicamente hayan hablado de su sexualidad, son pocas las instituciones deportivas que hayan desarrollado y puesto en práctica campañas de sensibilización, y las pocas asociaciones deportivas de gays y lesbianas en el deporte no tienen relevancia social. Así, a pesar de los avances y cambios sociales logrados, en la revisión de la literatura encontramos a un considerable número de autores que siguen confirmando la existencia un clima hostil a la diversidad de orientación sexual en el deporte (BARBERO, 2003, DAVIS-DELANO, 2014, GRIFFIN, 1998, O'BRIEN; SHOVELTON; LATNER, 2013, PRONGER, 2000), especialmente entre los deportistas más locales y menos populares (ANDERSON, 2002, STERNOD, 2011). Es decir, la existencia de un ambiente homófobo en el deporte, entendiéndose éste como el contexto donde domina el miedo irracional hacia las personas homosexuales (HYDE; DELAMATER, 2006) y que se puede expresar de diferentes formas.

Entre las diferentes pautas y acciones que generan marginación y que encontramos en el deporte se describen: estereotipos negativos; acoso verbal; discriminación en la selección del equipo; aislamiento social; pérdida de apoyos; y atención negativa de los medios 
(ALMEIDA; DERÓS; VON MÜHLEN, 2008, BARBER; KRANE, 2007, BRACKENRIDGE et al., 2007, LENSKYJ, 2014). Todo ello provoca que las personas de este colectivo se vean forzadas a permanecer invisibilizadas y silenciadas (DEVÍS; FUENTES; SPARKES, 2005, KRANE; BARBER, 2003, NORMAN, 2013). Como señalan Elling, De Knop, Knoppers (2003), esta opción de vida no es tanto una opción individual respetable, sino que a menudo representa una estrategia de supervivencia; al menos, hasta que su carrera deportiva acaba. Muchos de los que se mantienen escondidos huyen del estigma que supone ser señalado como homosexual. Este estigma homosexual está formado por tres aspectos diferentes de la homofobia (HEREK, 2009, PACHANKIS, 2007, VAN DE VEN; BORNHOLT; BAILEY, 1996), un aspecto cognitivo que hace referencia a las concepciones y creencias que se asumen sobre la homosexualidad, en muchos casos vinculados con estereotipos; un aspecto afectivo que está vinculado con los sentimientos hacia las personas o prácticas homosexuales como rechazo o incomodidad; por último el aspecto conductual que conecta con los comportamientos hacia gays y lesbianas, manifestándose en rechazos, insultos, e incluso agresiones.

En muchos casos, este clima de rechazo y persecución está promovida, o al menos consentida, por las instituciones. Casos como el de los últimos Juegos Olímpicos de Invierno en Sochi, Rusia en 2014 son un ejemplo de ello. La situación se mantuvo en el tiempo por la concomitancia de los organismos olímpicos y las federaciones que no hicieron mucho por cambiar el clima homofóbico del evento (LENSKYJ, 2014). Otro ejemplo del rechazo es la negativa del Comité Olímpico Internacional al uso del término Olímpico en los Gay Games (SYMONS, 2010). De igual modo, los medios de comunicación tienen un papel clave en la perpetuación de un clima deportivo poco tolerante con las minorías sexuales (WHANNEL, 2001). Además, muchos de los estudios realizados señalan que la situación es más complicada para hombres que para mujeres dada la diferente influencia de la heteronormatividad en el deporte femenino que masculino (ENG, 2006). Así, los estudios con jóvenes deportistas de Roper y Halloran (2007) y con técnicos de Ensign et al. (2011) señalan que las actitudes hacia gays y lesbianas eran peores entre los hombres que entre las mujeres. Este rechazo generalizado y evidente hacia gays y lesbianas en el deporte documentado en los trabajos anteriores, ha sido el que ha provocado que muchos de ellos antes de abandonar la práctica deportiva hayan buscando contextos más tolerantes y respetuosos donde sentirse más cómodos (WALTHER, 2006), como son los clubes, las ligas deportivas LGTB o los eventos internacionales como Gay Games o Outgames. En estos ambientes, los deportistas experimentan una sensación de mayor libertad, libres de prejuicios y discriminaciones (JARVIS, 2006, WATSON; TUCKER; DRURY, 2013, WELLARD, 2006), aunque se pueda caer fácilmente en la creación de guetos (SYMONS, 2007).

Sin embargo, también es verdad que aunque los estudios citados anteriormente señalan el 'lado oscuro' del deporte para gays y lesbianas, encontramos por otro lado estudios que en los últimos años rebaten 0 atenúan la persistencia de actitudes negativas en el deporte hacia las minorías sexuales. Así, el estudio de Kauer y Krane (2006) destaca que las mujeres que hicieron pública su homosexualidad, 'saliendo del armario' dentro del equipo femenino afectó positivamente a las actitudes de las jugadoras heterosexuales. También, el estudio longitudinal con jugadores de rugby y hockey hierba en Reino Unido de Anderson, Mccormack, Lee (2012), resalta la progresiva normalización en los últimos años entendiéndose como la reducción de las actitudes negativas hacia la homosexualidad entre los deportistas, haciéndose cada vez más visibles y siendo aceptados determinados comportamientos y prácticas que antes eran 
señalados y rechazados para los hombres. En la misma línea, los estudios de Ensign et al. (2011) y Oswalt y Vargas (2013) encuentran actitudes positivas hacia los deportistas gays entre los técnicos, aunque persistiendo un pequeño porcentaje de los entrenadores y entrenadoras con actitudes negativas. Estos cambios inclusivos, esta normalización de la homosexualidad que es percibida por los investigadores entre los deportistas y entrenadores, es también señalado por los estudios de Cashmore y Cleland (2012), Cleland ${ }^{1}$ y Nylund (2014) entre los fans y los medios de comunicación. Estos cambios no sólo se reportan con atletas gays: estudios recientes con deportistas lesbianas señalan en la misma dirección de cambio (ANDERSON; BULLINGHAM, ${ }^{2}$ STOELTING, 2011). Cada vez son más los agentes implicados en la creación de una cultura deportiva más tolerante: deportistas, técnicos, fans o periodistas. Sin embargo, todos los estudios resaltan la persistencia de pequeños colectivos que se mantienen con actitudes opuestas a la presencia de deportistas gays en las pistas.

En el caso de España, existen indicios de que la situación social de estos deportistas puede haber cambiado o estar cambiando. Sin embargo, la falta de estudios que analicen este problema en nuestra sociedad impide hacer una valoración objetiva y contrastada en un sentido u otro. Por eso, el objetivo que se plantea este trabajo es describir el discurso usado por jóvenes universitarios españoles a la hora de explicar la presencia de gays y lesbianas en el deporte.

\section{METODOLOGÍA}

\subsection{Técnicas y participantes}

Con el objeto de alcanzar el objetivo planteado se ha plateado el uso de la técnica de grupos de discusión o focus group. Como señalan Sparkes y Smith (2014) esta técnica es adecuada en estudios exploratorios pues permite un diálogo dinámico y constituye un espacio para validar experiencias en el tema de estudio. Estos diálogos y experiencias tienen una naturaleza discursiva por lo que el uso de metodologías cualitativas se antojan las más pertinentes.

Se organizaron durante el mes de noviembre de 2014 dos focus group independientes en el que tomaron parte un total de nueve estudiantes universitarios (cinco mujeres y cuatro hombres) de grados vinculadas al deporte: Ciencias de la Actividad Física y el Deporte (GCAFD) y Educación Primaria, Mención en Educación Física (GEP). La edad de los participantes estaba entre los 21 y los 23 años, y todos eran practicantes deportivos, desde la competición federada a la práctica recreativa. Una de ellos reconocía ser homosexual (Lorena), el resto se clasificaba como heterosexuales.

\subsection{Procedimiento}

De entre los estudiantes de las citadas titulaciones se presentó el estudio que se estaba realizando y se solicitaron voluntarios para participar en el estudio. De entre los voluntarios se seleccionaron finalmente, mediante muestreo de conveniencia, un total de nueve personas que

1 CLELAND, Jaime. Discussing homosexuality on association football fan message boards: A changing cultural context. International Review for Sociology of Sport, Otago. en prensa. doi:10.1177/1012690213475437 
conformaran una muestra estratificada en base al género (hombre y mujer), titulación (GCAFD y GEP) y orientación sexual (heterosexual y homosexual). Al total de participantes se les facilitó el consentimiento informado para su firma garantizándole la confidencialidad de sus respuestas y el tratamiento anónimo de los datos. Para garantizar aún más el anonimato los nombres de los participantes que aparecen en este trabajo son ficticios. Los dos grupos de discusión se desarrollaron de manera independiente, en un clima de respeto y confianza. Las entrevistas fueron conducidas por el autor de este trabajo, tuvieron una duración de entre 60 y 75 minutos, y fueron grabadas y posteriormente transcritas para su análisis.

\subsection{Análisis de los datos}

El análisis de las transcripciones se realizó con apoyo del software específico Atlas.ti 6.2. El uso de este tipo de herramientas permite un análisis más rápido y de mayor calidad de los datos (FLICK, 2014). Los datos se examinaron mediante análisis de contenido fragmentando las transcripciones en unidades de registro (ANDREU, 2008) con un significado en base a un sistema de categorías elaborado ad hoc fruto de la revisión de la literatura realizada previamente. A los participantes se les pedía que respondiera a una serie de preguntas sobre la situación de gays y lesbianas en el deporte, las reacciones de los aficionados, el contexto institucional, los estereotipos ligados a la homosexualidad, diferencias entre deportes... Los resultados que este trabajo presenta hacen mención solo a alguna de las categorías analizadas.

\section{RESULTADOS Y DISCUSIÓN}

Los resultados que se presentan en este trabajo son una muestra del trabajo global realizado, reflejando aquellos aspectos que tienen que ver con la progresiva visibilizacón de gays y lesbianas en el deporte, así como los discursos entorno a la homofobia en el deporte.

\subsection{No lo aceptan pero lo respetan}

Los análisis de las argumentaciones de este grupo de jóvenes participantes demuestran disparidad de posibilidades, desde un rechazo claro y contundente, hasta una aceptación de gays y lesbianas. Sin embargo, en la mayoría de los casos se destaca la polémica que genera la sexualidad de los deportistas, persistiendo en ese sentido un clima de rechazo a gays y lesbianas en el deporte, señalando que nuestra sociedad va con retraso con respecto con otras sociedades donde hay un clima de mayor tolerancia. Argumentan que a las personas les cuesta asimilar los cambios sociales que a un ritmo relativamente acelerado se están produciendo, por eso las primeras impresiones en estas situaciones son de rechazo.

Entrevistador: ¿Si alguien en el equipo dijera que es gay, creéis que lo aceptaría o que lo rechazarían?

Alberto: De todos no.

Lorena: Algunos sí, pero todos no.

En este sentido, la estudiante lesbiana expone su experiencia personal en un equipo durante los entrenamientos y los partidos: 
Lorena: ...simplemente no le gustan, no lo respetan o lo respetan en plan te hablo justamente lo necesario y vamos a jugar, y vamos practicar deporte para competir y ahí se acabó la relación. [...] A mí no, a mí no me resultó incómodo pero yo sé que hay gente que sí le puede... sí le puede molestar, o no lo puede...

Esta primera fase negativa de rechazo se superaría una vez pasado los primeros instantes según los jóvenes participantes. En este sentido, podemos encontrar varios estudios (ADAM; ANDERSON, 2012, ANDERSON, 2014, CUNNINGHAM; MELTON, 2012, MARGRATH; ANDERSON; ROBERTS ${ }^{3}$, ROPER; HALLORAN, 2007) que señalan que el clima hacia gays y lesbianas mejora cuando se entra en contacto directo con personas de estas minorías sexuales, creando de un clima más respetuoso e inclusivo, así como que un clima respetuoso facilita que los deportistas hagan pública su homosexualidad (CUNNINGHAM et al., 2014). Sin embargo, según los participantes este cambio no siempre lleva aparejado mayores niveles de aceptación, sino simplemente un cierto respeto quizás por miedo a ser señalado como homófobo/a,:

Lourdes: ...pero luego en el fondo no lo aceptan del todo. Es como que siempre hay un poco de rechazo, no sé, como que lo tratan de forma distinta, no lo integran del todo.

Como afirma en el siguiente fragmento Lidia, esta aparente aceptación no siempre lleva implícita una mayor visibilización de gays y lesbianas y está condicionada, siguiendo los patrones de la cultura don't ask, don't tell. En esta cultura la identidad de los deportistas gays no es tratada en igualdad con la heterosexual, llevando a gays y lesbianas a creer que no tienen derecho a hablar de su sexualidad (ANDERSON, 2002).

Lidia: ...si no lo muestras, pues bueno, te respeto porque nos olvidamos.

Estos ejemplos señalan una aceptación pública pero no siempre privada. Muchas personas se posicionan en lo políticamente correcto con gays y lesbianas, pero luego sus actitudes y opiniones son distintas, incluso opuestas. Podríamos señalar entonces que según apunta el discurso de los jóvenes, en España nos encontramos en lo que Anderson (2009) denomina: fase de 'homofobia cultural decreciente'. Es decir, coexisten distintas formas de entender las identidades de género, donde hay personas que no tienen miedo a transgredir los límites de la heteronormatividad, pero encontramos otras que siguen teniendo miedo a ser señalados como homosexuales por los problemas que ello acarrea. En este sentido, se destaca la necesidad de seguir trabajando, especialmente en el ámbito educativo, para eliminar estereotipos y superar prejuicios históricamente asentados en el imaginario colectivo.

Otros aspectos importantes para entender el clima de tolerancia hacia gays y lesbianas es el propio contexto social en el que se desarrolla. Anderson (2002) y Sternod (2011) señalan en sus trabajos que el deporte más local y menos popular suele ser especialmente propicio para producirse situaciones de rechazo.

Pablo: Yo creo que depende mucho del contexto sociocultural de la zona.

Paula: Es que volvemos a entrar en lo mismo, que depende del contexto sociocultural, que la educación que, por ejemplo, he recibido yo no es la misma que ha recibido Pablo. Depende de la educación parental, de los amigos, de la escuela. 
Los jóvenes entrevistados señalan la importancia de otros factores sociales como la edad de las personas. Los más mayores, por su formación y tradiciones, suelen ser más proclives a rechazar a los homosexuales:

Lorena: Claro, es que ahí entramos, estamos acostumbrados a lo que estamos acostumbrados y lo que llevamos toda la vida viendo, y en el momento que te cambia un poco el esquema ya... como que no gusta o hay un rechazo.

Mar: Yo, por ejemplo, creo que los jóvenes de hoy en día sí somos un poco más abiertos pero, por ejemplo, la generación de nuestros padres para arriba, creo que eso lo rechazarían.

Los jóvenes también son conscientes de las diferencias a la hora de afrontar la homosexualidad entre el deporte masculino y el femenino. En este sentido comentan que la situación de los hombres es más complicada (SKOGVANG; FASTING, 2013), pues tienen que romper con unos estereotipos asociados a la homosexualidad, que la heteronormatividad se ha encargado de difundir. La promiscuidad, la pederastia, el miedo a la agresión, son argumentos que los propios jóvenes utilizan para justificar sus creencias:

Luis: Pero yo creo que lo de la homosexualidad con los hombres está en plan más rechazado por la cosa tan simple de que como los hombres tenemos el pene [...] El hecho de que un hombre que es homosexual que nada más que sea más fuerte que tú te puede coger y te la puede meter doblada. En una mujer no, en una mujer no es tan fácil, tiene que haber un algo más.

Los argumentos que se muestran a continuación refuerzan las explicaciones de los trabajos de Eng (2006), Ensign et al. (2011) y Roper y Halloran (2007), pues coinciden en señalar a las mujeres como más tolerantes con gays y lesbianas.

Entrevistador: ¿Creéis que podría ser diferente una chica que sale del armario en un equipo femenino que un chico que sale del armario en un equipo masculino?

Lourdes: Las chicas podemos aceptar eso porque también tenemos entre nosotras... el contacto es más natural.

Lidia: Con hombres es peor yo creo.

A lo largo de las entrevistas con los jóvenes se extrae la idea de que el principal problema para gays y lesbianas son los conflictos (internos y externos) que se pueden crear haciendo pública su sexualidad. La más recurrente es el conflicto que se genera en los deportes colectivos dentro del grupo (GOUGH, 2007, JACOBSON, 2002). La homosexualidad de un deportista se convierte en factor generador de problemas entre los compañeros, con los aficionados y con los medios (BURY, 2012), incluso en clubes gay friendly (PRICE; PARKER, 2003).

Luis: yo creo que dentro de toda la afición que tiene el Real Madrid obviamente habría alguno que diría "¡Oh, el maricón este!, jmariquita!, jno queremos que juegue!, jvamos a perder por su culpa!"

Alex: Sí, por su afición, puede, pero la afición de la contra no.

Pablo: ...la afición contraria aprovecharía su homosexualidad para hacerle daño. Sería un constante "¡Maricón!, ¡Maricón!". De forma constante. Si es por ejemplo Guti, que tan solo tenía el pelo largo y cuidado, y era constantemente "¡Maricón!, ¡Maricón!" de la afición. Imagínate que sale del armario, pues ya se lo comen. 
Igualmente genera problemas internos, pues al hacer pública su homosexualidad, los deportistas corren el riesgo de perder el estatus que se han ganado como grandes deportistas (MOIOLI et al., 2014). Con todo ello, lo más conveniente es seguir con la política de muchos clubes de silenciar a los deportistas homosexuales (ANDERSON, 2011). Sin embargo, también podemos encontrar otros estudios donde los deportistas señalan que la 'salida del armario' de un compañero no debilitaría la cohesión del grupo (ADAMS; ANDERSON, 2012).

Lourdes: Es que yo creo que ya no es solo el jugador, sino que eso se tapa mucho por el equipo [...] Entonces yo creo que se tapa por eso, en plan de que la gente no sabe tratar esto, nos van a fusilar aquí a prensa y a lo mejor te puede crear a ti algún tipo de trauma psicológico, pues ¿para qué lo vas a decir? ¿Sabes? Si no vas a tener... no va a ser algo grato, al revés, puede tener muchas consecuencias.

Luis: $O$ que tu entrenador te diga algo en plan "No digas nada, que me parece muy bien, pero no digas nada no vaya a ser que ahora te metan aquí la prensa... yo qué sé... y no queremos".

En definitiva, según los discursos de los jóvenes, el proceso de normalización de lesbianas y gays en el deporte pasa por un primer proceso de rechazo generalizado. Este rechazo se suaviza cuando hablamos de mujeres y de la generación más reciente, pues según los participantes presentan actitudes de mayor tolerancia gracias a la educación que han recibido. Pasado este primer paso, muchas de las personas consienten un trato más cercano y directo con los homosexuales, respetando sus características, pero dicho respeto no siempre conllevan una aceptación de estas personas en el deporte, manteniendo constantemente una cierta lejanía. Como señalan Penna y Sánchez-Sainz (2015), esto no significa una reducción de la homofobia, sino que se han modificado sus formas de presentación y que a continuación desgranaremos.

Las situaciones de mayor conflicto se desencadenan desde el punto de vista de los participantes, en los problemas que desencadena hacer pública la sexualidad de un deportista. Los jóvenes señalan que un deportista al hacer esto está generando una situación de conflicto con sus compañeros, con los medios y con los aficionados. Por ello argumentan que son los propios clubes los que no animan, e incluso ocultan la orientación sexual de alguno de sus jugadores, a temor de las consecuencias que pueda tener.

\subsection{De la incomodidad a la agresión. Diferentes expresiones de la homofobia en el deporte}

Una vez analizada las creencias de los jóvenes con respecto al nivel de aceptación de gays y lesbianas en el deporte, esta segunda parte del trabajo presenta las opiniones y argumentaciones de los jóvenes con respecto a las maneras de manifestarse y aflorar el discurso homófobo. Siguiendo la línea argumental que platean los estudios de Herek, (2009), Pachankis, (2007) y Van de Ven, Bornholt, y Bailey (1996), hemos dividido la homofobia para su estudio (aunque es complicado independizarlas completamente unas de otras) en tres maneras de exteriorizarse: cognitiva, afectiva y conductual.

Homofobia Cognitiva. Esta primera manifestación de la homofobia que se presenta tiene que ver con la representación de la homosexualidad en el ideario y en las creencias de las personas. Los estereotipos que la sociedad heteronormativa ha construido en torno a gays y lesbianas es determinante a la hora de configurar dicho pensamiento. Como hemos comentado 
anteriormente, esta homofobia cognitiva tiene un mayor peso dentro la homosexualidad masculina y el deporte. El deporte, al considerarse bastión de la heteronormatividad, privilegia un tipo de masculinidad hegemónica (CONNELL, 1995, MESSNER, 1992), marginando otros tipos de masculinidades no hegemónicas (PARKER; CURTNER-SMITH, 2012).

En este sentido, los jóvenes son claros. Los deportistas varones (homosexuales o no) que no se ajustan a los patrones de la masculinidad hegemónica (CONNELL, 1995) son especialmente rechazados. El aspecto físico y la actitud deportiva son dos factores determinantes según los jóvenes entrevistados para tener éxito en el deporte; la musculatura, la agresividad o la valentía son características inherentes y necesarias del deporte. Así, los gays, que en muchos casos no se amoldan a los cánones hegemónicos y dominantes de la masculinidad son rechazados, en base a esa visión estereotipada del deporte.

Luis: [Hablando de la película Summer Storm] ...los flacuchitos. Claro, esos dos, sobre todo en la escena esta de "es que me da miedo ir", "no pasa nada, yo te protejo con esto"... ¿Dónde vas illo? [Ríe] ¿Dónde vas? Yo creo que eso, dentro de un equipo, perjudicaría más que el gay este que era más normalito, o el más fuerte. Mira, me da más confianza, a lo mejor sus aptitudes físicas son mejores, puedo confiar más en él a la hora de...

Lourdes: Es que además las personas que se sienten incómodas con eso se sienten más cómodas con una persona homosexual que no lo demuestra o que no se le nota tanto entre comillas, que otro que sí [...] dice que los hombres que son homosexuales que son tipo Boris [lzaguirre] le dan más asco que una persona como Jaime Cantizano. [...] él es gay pero no se le nota", es un hombre, y luego está la típica "maricona loca.

Otros estereotipos presentes en el discurso de los jóvenes sobre la homosexualidad no están vinculados directamente con el deporte, pero sin embargo le afectan directamente. Así prejuicios como que los homosexuales son más promiscuos que los heterosexuales, que hay vinculación entre homosexualidad y pederastia... son ideas que los jóvenes reconocen como erróneas pero que siguen dominando el ideario de muchas personas en nuestra sociedad, influyendo en los comportamientos. En este sentido, Lorena, la joven lesbiana, en base a su propia experiencia señala:

Lorena: Es que la homosexualidad se ve... yo por comentarios que he escuchado ahora de gente, de que, por ejemplo, mi amiga es lesbiana y cuando se enteró esa chavala fue como: ya no duermo en el mismo cuarto que ella, ni entro en el mismo probador. En cambio, con un tío sí dormía. Entonces estamos en las mismas, se cree que por ser homosexual le va a gustar todas las tías del mundo. [...] Es como que está relacionado, si eres homosexual es que te gusta to' dios, te gustan todos los tíos si se te acerca un tío.

Homofobia Afectiva. Como acabamos de ver, la consecuencia de asumir muchos de los estereotipos sobre la homosexualidad es que se generan en las personas sentimientos de rechazo e incomodidad. El asco ante escenas de intimidad entre dos gays, la preocupación ante la presencia de un homosexual en el vestuario, son reacciones todavía habituales en nuestra sociedad según los participantes. El deporte, especialmente los deportes de equipo, por sus características puede despertar potencialmente muchas de estas sensaciones entre los deportistas y así lo señalan los jóvenes en base a sus experiencias:

Mar: Sí, lo que pasa es que se dice que los hombres tienen que ser más fuertes, no pueden llorar, no seas una nena. Entonces, claro, al decir, a lo mejor "soy gay" o "soy 
homosexual" a lo mejor ya tiende... [...] O por ejemplo, está en el vestuario y se sienten incómodos porque hay un gay, porque dice a lo mejor... yo eso, por ejemplo, eso yo por ejemplo lo he escuchado y eso yo creo que no tiene por qué ¿sabes?

Estos sentimientos generados son también señalados por otros trabajos precedentes como el de Griffin et al. (2002), o los de Dashper (2012) en deportes ecuestres, y Adams y Anderson (2012) y Drury (2011) en fútbol. Situación que además se acentúa cuando se tratan de clubes, como se ha señalado en la introducción, que acogen con tolerancia y de manera pública a gays y lesbianas:

Lidia: [Hablando de clubes gay friendly] ...que sea todo el equipo entero gay. Yo creo que eso sí echaría para atrás.

Mar: Yo creo que puede ser un poco más incómodo.

Homofobia Conductual. Este tipo de actitud homófoba es la que más ha cambiado en los últimos años. Gracias a los cambios legislativos el número de agresiones físicas hacia personas homosexuales ha bajado con respecto a años atrás. Sin embargo, como señala el informe del Ministerio del Interior sobre Evolución de los delitos de odio en España (ESPANHA, 2013), los delitos más denunciados en España son aquellos que tienen que ver con la orientación sexual de las personas, muchos de ellos acaecidos en campos deportivos. En este mismo sentido, otros informes específicos del deporte, como el desarrollado por la asociación escocesa Equality Network en 2012 señalaron que la mitad de los encuestados había sido testigo de comportamientos homofóbicos, y que 98\% habían sido de tipo verbal. Así, los jóvenes reconocen esta realidad:

Lidia: Es que, aunque te aceptan siempre suele haber bromas, siempre suele haber comentarios. Aunque hasta tus amigos te acepten... siempre es como... no sé, tienen en cuenta que eres homosexual ¿no?

Lourdes: El hombre, a lo mejor, sí hace más diferenciaciones, o hace bromas que pueden molestar al otro, herir su sensibilidad o... aunque no sea queriendo, pero yo creo que sí se da.

Los comportamientos más frecuentes dentro del ámbito del deporte son según se desprende de las opiniones de los jóvenes, el uso del lenguaje como herramienta para atacar a las minorías sexuales (CASHMORE; CLELAND, 2011, CAUDWELL, 2011; DICK, 2009), aunque en ocasiones este comportamiento no sea percibido como discriminativo por los propios autores.

Entrevistador: Después de haber salido del armario, en el Bernabéu, ¿qué ganarían, los pitidos o los aplausos?

Pablo: ...la afición contraria aprovecharía su homosexualidad para hacerle daño.

Luis: Eso es cierto.

Pablo: Sería un constante "¡Maricón!, ¡Maricón!”. De forma constante. Si es por ejemplo Guti, que tan solo tenía el pelo largo y cuidado, y era constantemente "¡Maricón!, ¡Maricón!" de la afición. Imagínate que sale del armario, pues ya se lo comen.

Este tipo de conductas generalizadas son consideradas por los deportistas como una de las principales barreras para 'salir del armario' (BURY, 2012). Así pues entendemos que clubes e instituciones deportivas, que teniendo la posibilidad de arbitrar medidas, han de 
trabajar por mejorar el clima de respeto y tolerancia en los estadios, planteando medidas para eliminar los abusos (verbales y no verbales) en los terrenos de juego.

\section{CONCLUSIONES}

El objetivo principal de este estudio ha sido describir el discurso usado por jóvenes universitarios españoles a la hora de explicar la presencia de gays y lesbianas en el deporte. Para ello, la técnica de focus group empleada con un grupo de jóvenes universitarios ha permitido extraer la manera en que estos jóvenes explican la realidad de gays y lesbianas en el deporte español.

Los resultados del trabajo destacan la presencia de un clima de respeto inicial en el deporte, con mayor intensidad en el caso de mujeres jóvenes. Sin embargo, el suavizado de la homofobia posterior es más sutil, desapareciendo las actitudes de rechazo claro y público, pero persistiendo actitudes de rechazo que tienen más que ver con la homofobia afectiva y cognitiva. El uso del lenguaje y las bromas sigue apareciendo como el instrumento más común a la hora de señalar a gays y lesbianas en los estadios. El miedo a los conflictos que se pueden generar en la propia persona y en el grupo es la principal causa de permanecer la homosexualidad como un tema tabú en el deporte.

Las características del estudio han permitido obtener una serie de resultados interesantes para el estudio de la situación de gays y lesbianas en el deporte español. Sin embargo, este mismo diseño del trabajo presentado conlleva una serie de limitaciones que a continuación resaltamos. Por un lado, a pesar de que la conformación de la muestra ha sido heterogénea (hombres y mujeres; heterosexuales y homosexuales) el reducido tamaño de la muestra, con solo diez participantes reduce la potencia de los resultados pues además en ella solo figuran jóvenes con formación universitaria. Es bastante probable que las opiniones y creencias cambiasen si en el estudio hubiesen participado personas de diferentes edades y niveles educativos. Otra limitación del diseño del estudio, pero que son inherentes al uso de las metodologías cualitativas, es la imposibilidad de generalizar los datos obtenidos. Teniendo en cuenta las limitaciones del estudio, y siendo conscientes del vasto trabajo que queda por hacer para alcanzar la igualdad, recomendamos a modo de prospectiva continuar con el estudio de la situación de las minorías sexuales en el deporte en España, presentando trabajos que combinen metodologías de corte cualitativo y cuantitativo, a fin de realizar un análisis de la realidad más exhaustivo y eficiente.

\section{REFERENCIAS}

ADAMS, Adi; ANDERSON, Eric. Exploring the relationship between homosexuality and sport among the teammates of a small Midwestern Catholic College soccer team. Sport, Education and Society, London, v. 17, n. 3, p. 347-363, 2012.

ALMEIDA, Thaís R.; DERÓS, Carolina; VON MÜHLEN, Johanna. A ofensa, o juiz e a sentença: Gênero e sexualidade em jogo no futebol brasileiro. In: FAZENDO GÊNERO 8: CORPO, VIOLÊNCIA E PODER, Florianópolis, 2008. Anais... Disponible en: < http://www. fazendogenero.ufsc.br/8/sts/ST67/Almeida_Deros_Muhlen_67.pdf >. Acceso en: 21 feb. 2015. 
ANDERSON, Eric. Openly gay athletes: contesting hegemonic masculinity in a homophobic environment. Gender \& Society, Kansas, v. 16, n. 6, p. 860-877, 2002.

ANDERSON, Eric. Inclusive masculinity: the changing nature of masculinities. London: Routledge, 2009.

ANDERSON, Eric. Updating the outcome. Gay athletes, straight teams, and coming out in educationally based sport teams. Gender \& Society, Kansas, v. 25, n. 2, p. 250-268,

ANDERSON, Eric. 21st Century Jocks: sporting men and contemporary heterosexuality. London: Palgrave Macmillan, 2014.

ANDERSON, Eric; MCCORMACK, Mark; LEE, Harry. Male Team Sport Hazing Initiations in a Culture of Decreasing Homohysteria. Journal of Adolescent Research, Florida, n. 27, p. 427448, 2012.

ANDREU, Jaime. Las técnicas de análisis de contenido: una revisión actualizada. Granada: Universidad de Granada, 2008.

AYVAZO, Shiri; SUTHERLAND, Sue. Uncovering the secrets: Homophobia in Physical Education. Action in Teacher Education, Virginia, v. 31, n. 3, p. 56-69, 2009.

BARBER, Heather; KRANE, Vikki. Creating Inclusive and Positive Climates in Girls' and Women's Sport: Position Statement on Homophobia, Homonegativism, and Heterosexism. Women in Sport \& Physical Activity Journal, Texas, v. 16, n. 1, p. 53-56, 2007.

BARBERO, José Ignacio. La educación física y el deporte como dispositivos normalizadores de la heterosexualidad. In: GUASCH, Oscar; VIÑUALES, Olga (Ed.). Sexualidades: diversidad y control social. Barcelona: Bellaterra, 2003. p. 355-377.

BRACKENRIDGE, Celia et al. Driving down participation: homophobic bullying as a deterrent to doing sport. In: AITCHISON, Cara C. (Ed.). Sport \& gender identities: masculinities, femininities and sexualities. London: Routledge, 2007. p. 122-139.

BURY, Jonah. Can a footballer be gay? The problem of (homo)sexuality and coming out in English professional football. In: ADAMS, Andrew; SPRACKLAN, Karl (Ed.). Sport and leisure ethics, rights and social relationships. Eastbourne: Leisure Studies Association, 2012. p. 7995.

CASHMORE, Ellis; CLELAND, Jaime. Glasswing butterflies: gay professional football players and their culture. Journal of Sport and Social Issues, Massachusetts, v. 35, n. 4, p. 420-436, 2011.

CASHMORE, Ellis; CLELAND, Jaime. Fans, homophobia and masculinities in association football: evidence of a more inclusive environment. The British Journal of Sociology, London, v. 63, n. 2, p. 370-387, 2012.

CAUDWELL, Jayne. Does your boyfriend know you're here? the spatiality of homophobia in men's football culture in UK. Leisure Studies, London, v. 30, n. 2, p. 123-138, 2011.

CLELAND, Jaime. Racism, Football Fans, and Online Message Boards: How Social Media Has Added a New Dimension to Racist Discourse in English Football. Journal of Sport \& Social Issues, Massachusetts, v. 38, n. 5, p. 415-431, 2014. 
CUNNINGHAM, George B.; MELTON, Nicole. prejudice against lesbian, gay, and bisexual coaches: the influence of race, religious fundamentalism, modern sexism, and contact with sexual minorities. Sociology of Sport Journal, Minnesota, v. 29, n. 3, p. 283-305, 2012.

CUNNINGHAM, George B. et al. Psychological safety and the expression of sexual orientation and personal identity. In: HARGREAVES, Jennifer; ANDERSON, Eric (Ed.). Routledge handbook of sport, gender and sexuality. London: Routledge, 2014. p. 406-415.

DASHPER, Katherine. Dressage in full of queens! masculinity, sexuality and equestrian sport. Sociology, Oxford, v. 46, n. 6, p. 1109-1124, 2012.

DAVIS-DELANO, Laurel R. Sport as context for the development of women's same-sex relationships. Journal of Sport \& Social Issues, Massachusetts, v. 38, n. 3, p. 263-285, 2014.

DEVÍS, Jose Devis; FUENTES, Jorge; SPARKES, Andrew C. ¿Qué permanece oculto del currículum oculto? Las identidades de género y de sexualidad en la educación física. Revista Iberoamericana de Educación, Madrid, n. 39, p. 73-90, 2005.

DICK, Sam. Leagues behind-football's failure to tackle anti-gay abuse. London: Stonewall, 2009.

DOWLING, Fiona. Teacher educators' gendered workplace tales. In: PFISTER, Gertrud; SISJORD, Mari Kristin (Ed.). Gender and Sport. Changes and Challenges. Münster: Waxmann, 2013. p. 217-231.

DRURY, Scarlett. It seems really inclusive in some ways, but... inclusive just for people who identify as lesbian': discourses of gender and sexuality in a lesbian-identified club. Soccer \& Society, London, v. 12, n. 3, p. 421-442, 2011.

ELLING, Agnes; DE KNOP, Paul; KNOPPERS, Annelies. Gay/lesbian sport clubs and events: places of homo-social bonding and cultural resistance? International Review for the Sociology of Sport, Warsaw, v. 38, n. 4, p. 441-456, 2003.

ENG, Heidi. Queer athletes and queering in sport. In: CAUDWELL, Jayne (Ed.). Sport, sexualities and queer/theory. London: Routledge, 2006. p. 46-61.

ENSIGN, Kristine et al. Athletic trainers' attitudes toward lesbian, gay, and bisexual National Collegiate Athletic Association student-athletes. Journal of Athletic Training, Kansas, v. 46, n. 1, p. 69-75, 2011.

EQUALITY NETWORK. Out of sport: tackling homophobia and transphobia in sport. Edinburgh: Equality Network, 2012. Disponible en: <http://www.equality-network.org/wp-content/ uploads/2013/03/Out-for-Sport-Report.pdf>. Acceso en: 21 feb. 2015.

ESPANHA. Ministerio del Interior. Informe sobre la evolución de los delitos de odio. Madrid: Gabinete de Coordinación y Estudios, 2013. Disponible en: <http://explotacion.mtin.gob.es/ oberaxe/documentacion descargaFichero?bibliotecaDatold=4063>. Acceso en: 22 feb. 2015

FLICK, Uwe. An introduction to qualitative research. 5. ed. London: SAGE, 2014.

GOUGH, Brendan. Coming out in the heterosexist world of sport: a qualitative analysis of web posting by gay athletes. Journal of Gay \& Lesbian Psychotherapy, Philadelphia, v. 11, n. 1-2, p. 153-174, 2007.

GRIFFIN, Pat. Strong women, deep closet: Lesbian and homophobia in sport. Champaign, IL: Human Kinetics, 1998. 
GRIFFIN, Pat et al. It takes a team! making sports safe for lesbian, gay, bisexual and transgender athletes and coaches. an education kit for athletes, coaches, and athletic directors. New York: Women's Sports Fundation, 2002.

GRIFFIN, Pat. LGTB equality in sports: celebrating our successes and facing our challenges. In: CUNNINGHAM, George (Ed.). Sexual orientation and gender identity in sport. Texas: A\&M University, 2012. p. 1-12.

HYDE, Janet.; DELAMATER, John. Understanding human sexuality. 9 ed. Boston: McGrawHill, 2006.

HEREK, Gregory M. Sexual stigma and sexual prejudice in the United States: A conceptual framework. In: HOPE, Debra A. (ed.). Contemporary perspectives on lesbian, gay, and sexual identities. New York, NY: Springer, 2009. p. 65-111.

JACOBSON, Jennifer. The loneliest athletes. Chronicle of Higher Education, Washington, D.C., v. 11, n. 1, p. A33-A34, 2002.

JARVIS, Nigel. Ten men out: gay sporting masculinities in softball. In: CAUDWELL, Jayne (Ed.). Sport, sexualities and queer/theory. London: Routledge, 2006. p. 62-75.

KAUER, Kerrie; KRANE, Vikki. "Scary dykes" and "Feminine Queens": Stereotypes and Female Collegiate Athletes. Women in Sport \& Physical Activity Journal, Texas, v. 15, n. 1, p. 42-55, 2006.

KRANE, Vikki; BARBER, Heather. Lesbian experiences in sport: a social identity perspective. Quest, Champaign, n. 55, p. 328-346, 2003.

LENSKYJ, Helen. Sexual diversity and the Sochi 2014 Olympics. Basingstoke: Palgrave Macmillan, 2014.

MESSNER, Michael A. Power at play: sports and the problem of masculinity. Boston: Beacon, 1992.

MOIOLI, A. et al. Soccer and homosexuality: The conflicts that lie within the affective game of the coach-adolescent athlete relationship. Motriz, Rio Claro, v. 20, n. 4, p. 346-358, 2014.

NORMAN, Leanne. The concepts underpinning everyday gendered homophobia based upon the experiences of lesbian coaches. Sport in Society, London, v. 16, n. 10, p. 1326-1345, 2013.

NYLUND, David. Transmitting softer masculinity. Sports talk radio and masculinity. In: HARGREAVES, Jennifer; ANDERSON, Eric (Ed.). Routledge Handbook of Sport, Gender and Sexuality. London: Routledge, 2014. p. 453-460.

O'BRIEN, Kerry S.; SHOVELTON, Heather; LATNER, Janet D. Homophobia in physical education and sport: The role of physical/sporting identity and attributes, authoritarian aggression, and social dominance orientation. International Journal of Psychology, Montreal, v. 48, n. 5 , p. 891-899, 2013.

OSWALT, Sara B.; VARGAS, Tiffanye M. How safe is the playing field? Collegiate coaches' attitudes towards gay, lesbian, and bisexual individuals. Sport in Society, London, v. 16, n. 1, p. 120-132, 2013.

PACHANKIS, John E. The psychological implications of a concealing a stigma: a cognitiveaffective-behavioral model. Psychological Bulletin, Washington, D.C., v. 133, n. 2, p. 328-345, 2007. 
PARKER, Mitchum B.; CURTNER-SMITH, Mathew D. Sport education: a panacea for hegemonic masculinity in physical education or more of the same? Sport, Education and Society, London, v. 17, n. 4 , p. $479-496,2012$.

PENNA, Melani; SÁNCHEZ-SÁINZ, Mercedes. Evaluación de la homofobia en los futuros docentes de Educación Secundaria. Revista de Investigación Educativa, Barcelona, v. 33, n. 1, p. 83-98, 2015.

PRICE, Michael; PARKER, Andrew. Sport, sexuality, and the gender order: amateur rugby union, gay men, and social exclusion. Sociology of Sport Journal, Minnesota, n. 20, p. 108-126, 2003.

PRONGER, Brian. Homosexuality and sport. Who's winning? In: MCKAY, J.; MESSNER, M.; SABO, D. (Ed.). Masculinities, gender relations, and sport. London: SAGE, 2000. p. 222-244.

ROPER, Emily A.; HALLORAN, Erin. Attitudes towards gay men and lesbians among heterosexual male and female student-athletes. Sex Roles, Pittsburgh, n. 57, p. 919-928, 2007.

SKOGVANG, Bente O.; FASTING, Kari. Football and sexualities in Norway. Soccer \& Society, London, v. 14, n. 6, p. 872-886, 2013.

SPARKES, Andrew C.; SMITH, Brett. Qualitative research methods in sport, exercise and health. London: Routledge, 2014.

STERNOD, Brandon. Come out to play. Confronting homophobia in sports. In: SPICKARD, Sandra; LAMPMAN, Brian (Ed.). Learning culture through sports: perspective on society and organized sports. Landham, MD: Rowman and Littlefield, 2011. p. 92-106.

STOELTING, Suzanne. Disclosure as an Interaction: Why Lesbian Athletes Disclose Their Sexual Identities in Intercollegiate Sport. Journal of Homosexuality, Philadelphia, v. 58, n. 9, p. 1187-1210, 2011.

SYMONS, Caroline. The gay games: a history. New York: Routledge, 2010.

SYMONS, Caroline. Challenging homophobia and heterosexism in sport: the promise of the Gay Games. In: AITCHISON, Cara Carmichael (Ed.). Sport \& Gender Identities: masculinities, femininities and sexualities. London: Routledge, 2007. p. 140-159.

VAN DE VEN, Paul; BORNHOLT, Laurel; BAILEY, Michael. Measuring cognitive, affective, and behavioral of homophobic reaction. Archives of Sexual Behavior, New York, v. 25, n. 2, p. 155179, 1996.

WALTHER, Tanja. Kick it out: homophobia in football. Amsterdam: European Gay and Lesbian Sport Federation, 2006.

WATSON, Rebecca; TUCKER, Lee; DRURY, Scarlett. Can we make a difference? examining the transformative potential of sport and active recreation. Sport in Society, New York, v. 16, n. 10, p. 1233-1247, 2013.

WELLARD, lan. Exploring the limits of queer and sport: gay men playing tennis. In: CAUDWELL, Jayne (Ed.). Sport, sexualities and queer/theory. London: Routledge, 2006. p. 76-89.

WHANNEL, Garry. Media sport stars: masculinities and moralities. London: Routledge, 2001. 